\title{
Study of deconfined quark matter at zero temperature and high density
}

N. Yu. Astrakhantsev, ${ }^{a b}$ V. G. Bornyakov, ${ }^{b c d}$ V. V. Braguta, ${ }^{a b d e}$ E.-M. Ilgenfritz, ${ }^{e}$

A. Yu. Kotov, ${ }^{a b e}$ A. A. Nikolaev ${ }^{* b d}$ and A. Rothkopf ${ }^{f}$

${ }^{a}$ Moscow Institute of Physics and Technology

141700 Dolgoprudny, Russia

${ }^{b}$ Institute for Theoretical and Experimental Physics, NRC “Kurchatov Institute”

117218 Moscow, Russia

c Institute for High Energy Physics, NRC “Kurchatov Institute"

142281 Protvino, Russia

${ }^{d}$ Far Eastern Federal University, School of Biomedicine 690950 Vladivostok, Russia

e Bogoliubov Laboratory of Theoretical Physics, Joint Institute for Nuclear Research 141980 Dubna, Russia

${ }^{f}$ Faculty of Science and Technology, University of Stavanger NO-4036 Stavanger, Norway

E-mail: nikita.astrakhantseveitep.ru, braguta@itep.ru,

ilgenfri@theor.jinr.ru, kotov@itep.ru, Alexander.Nikolaeveitep.ru,

alexander.rothkopfeuis.no

We study the interactions among a static quark-antiquark pair in the presence of dense two-color quark matter within lattice simulations. To this end we compute Polyakov line correlation functions and determine the renormalized color-averaged, color-singlet and color-triplet grand potentials. The color-singlet grand potential allows us to elucidate the number of quarks induced by a static quark antiquark source, as well as the internal energy of such a pair in dense quark matter. We furthermore determine the screening length, which in the confinement phase is synonymous with the string breaking distance. The screening length is a decreasing function of baryon density, due to the possibility to break the interquark string via a scalar diquark condensate at high density. We also study the large distance properties of the color singlet grand potential in a dense medium and find that it is well described by a simple Debye screening formula, parameterized by a Debye mass and an effective coupling constant. The latter is of order of unity, thus even at large density two-color quark matter is a strongly correlated system.

PACS: $12.38 . \mathrm{GC}, 12.38 . \mathrm{Aw}$

XIII Quark Confinement and the Hadron Spectrum - Confinement 2018

31 July - 6 August 2018

Maynooth University, Ireland

${ }^{*}$ Speaker. 


\section{Introduction}

Knowledge of the properties of QCD at large baryon density is needed to interpret the results of heavy ion collisions experiments. In particular, this is the case at the future experiments of NICA (JINR, Dubna) and FAIR (Darmstadt, Germany), which are designed to study the region of high baryon density. Input from the theory side is hence urgently needed. An understanding of the properties of matter in the corresponding region of the QCD phase diagram is also extremely important in astrophysics, for example, for a correct description of the fusion of neutron stars.

An interesting area of finite temperature lattice simulations is the study of the interaction between a quark-antiquark pair and the interaction of the pair with the QCD medium (see e.g. [1, $2,3,4]$ ). The in-medium properties of QCD are prominently encoded in the correlation function of Polyakov loops. The Polyakov loop correlator is directly related to the free energy of the inmedium quark-antiquark pair. In the confinement phase the free energy extracted is known to be a linear increasing function at intermediate distances. It is known that at zero temperature the QCD string breaks at distances above $1.2 \mathrm{fm}$ [5]. On the other hand in the deconfinement phase at large distances the free energy also flattens off, the reason being a screening of the interactions between the quark and antiquark due to liberated colored medium degrees of freedom. The question of whether or how the screening properties of QCD may be captured by an analogous and equally simple Debye screening formula in analogy with the Abelian theory is a ongoing field of research. The properties of the correlation function of Polyakov loops in finite temperature QCD have been thoroughly studied in lattice simulations [1, 2, 3, 4]. More recently the Polyakov loop correlator on the lattice has been compared to effective field theory predictions, both in a perturbative setting in pNRQCD and perturbatively matched EQCD [6], also in analytic studies [7, 8].

While it is an interesting proposition to carry out similar studies of the Polyakov loop at finite baryon density in QCD, the usual methods of lattice QCD unfortunately break down because of the so-called sign problem and so far only results for small values of the baryon chemical potential were obtained [9]. Instead of pursuing the question of finite density physics in QCD directly, we here turn to the study of theories, which are similar to QCD but are not plagued by the sign problem. We believe that in particular the study of dense two-color QCD [10,11] allows us to learn about the properties of three-color QCD at non-zero chemical potential. Other candidate theories not further pursued here are e.g. QCD at nonzero isospin chemical potential $[12,13]$. We can not expect to obtain quantitative predictions from such a strategy, while vital qualitative insight may be gained.

In this paper we continued our study of $\mathrm{SU}(2) \mathrm{QCD}$ at finite density and low temperature based on lattice simulations. Our simulations were performed on $32^{4}$ lattices with rooted staggered fermions at a relatively small lattice spacing $a=0.044 \mathrm{fm}$, which allowed us to study two-color QCD at very large baryon densities (up to quark chemical potential $\mu_{q}>2000 \mathrm{MeV}$ ) while avoiding strong lattice artifacts. Technical details of the simulations may be found in [14].

\section{The grand potential of a static quark-antiquark pair in dense quark matter}

The grand potential $\Omega_{\bar{q} q}\left(r, \mu_{q}\right)$ of a static quark-antiquark pair placed within a distance of $r$ 
into the dense medium can be represented in terms of the correlator of Polyakov loops

$$
\frac{\Omega_{\bar{q} q}\left(r, \mu_{q}\right)}{T}=-\log \left\langle\tilde{\operatorname{Tr}} L_{\vec{x}} \tilde{\operatorname{Tr}} L_{\vec{y}}^{\dagger}\right\rangle+c\left(\mu_{q}\right), r=|\vec{x}-\vec{y}|,
$$

where $\tilde{\mathrm{Tr}}=\frac{1}{2} \operatorname{Tr}$ and the Polyakov loop is given as the trace of a product of gauge links in temporal direction $L_{\vec{x}}=\prod_{\tau=0}^{N_{\tau}-1} U_{\mu=0}(\vec{x}, \tau)$. The quantity $c\left(\mu_{q}\right)$ denotes a divergent renormalization constant, which is related to the self-energy of a quark or antiquark source. In the limit $r \rightarrow \infty$, the correlation between the Polyakov lines becomes negligible and the grand potential $\Omega_{\infty}\left(\mu_{q}\right)$ is given by the squared expectation value of the volume-averaged Polyakov loop, $\langle L\rangle=\left\langle N_{s}^{-3} \sum_{\vec{x}} \tilde{\operatorname{Tr}} L_{\vec{x}}\right\rangle$ :

$$
\frac{\Omega_{\infty}\left(\mu_{q}\right)}{T}=\frac{1}{T} \lim _{r \rightarrow \infty} \Omega_{\bar{q} q}\left(r, \mu_{q}\right)=-\log |\langle L\rangle|^{2}+c\left(\mu_{q}\right) .
$$

In pure gauge theory the expectation value of the Polyakov line, which is defined as $L^{r e n}\left(\mu_{q}\right)=\exp \left(-\Omega_{\infty}\left(\mu_{q}\right) / 2 T\right)$, is the order parameter of the confinement/deconfinement transition, but not in the case of dynamical quarks in the simulations. However, one can interpret the $\Omega_{\infty}\left(\mu_{q}\right) / 2$ as the grand potential of one quark or one antiquark in dense quark matter. Thus one may expect that in the confined phase $\Omega_{\infty}\left(\mu_{q}\right)$ is much larger than that in the deconfined phase.

The color-singlet grand potential $\Omega_{1}\left(r, \mu_{q}\right)$ is defined as

$$
\frac{\Omega_{1}\left(r, \mu_{q}\right)}{T}=-\log \left\langle\tilde{\operatorname{Tr}}\left(L_{\vec{x}} L_{\vec{y}}^{\dagger}\right)\right\rangle+c^{\prime}\left(\mu_{q}\right) .
$$

Notice that this potential is not gauge invariant, so, in order to calculate $\Omega_{1}\left(r, \mu_{q}\right)$ one has to fix the gauge. In this study we choose conventionally the Coulomb gauge. Also color-singlet, color averaged grand potential $\Omega_{\bar{q} q}\left(r, \mu_{q}\right)$ and color-triplet grand potential $\Omega_{3}\left(r, \mu_{q}\right)$ are constrained by the following relation [15] (note that $N_{c}=2$ ):

$$
\exp \left(-\frac{\Omega_{\bar{q} q}\left(r, \mu_{q}\right)}{T}\right)=\frac{1}{4} \exp \left(-\frac{\Omega_{1}\left(r, \mu_{q}\right)}{T}\right)+\frac{3}{4} \exp \left(-\frac{\Omega_{3}\left(r, \mu_{q}\right)}{T}\right) .
$$

It is also worth to note that at short distances $\left(r \mu_{q} \ll 1\right)$ the running of the coupling constant is determined by the scale $\sim 1 / r$, and the influence of the chemical potential on the running coupling can be neglected. The perturbative one-gluon exchange expression for the grand potentials at short distances has the form $\Omega_{1}\left(r, \mu_{q}\right)=-3 \Omega_{3}\left(r, \mu_{q}\right)+\mathscr{O}\left(g^{4}\right)=-\frac{g^{2}(r)}{8 \pi r}+\mathscr{O}\left(g^{4}\right)$.

To perform the renormalization of the grand potentials we follow the procedure proposed in $[1,2,3]$, adopted here for the calculation at finite density. We fix the renormalization constant $c^{\prime}\left(\mu_{q}\right)$ through the matching condition for $\Omega_{1}\left(r, \mu_{q}\right)$ at short distances to the short distance behavior of the interaction potential $V(r)$. The renormalization for the grand potential $\Omega_{\bar{q} q}\left(r, \mu_{q}\right)$ can be fixed using matching at large distances $r$, where the color averaged and the color singlet grand potentials are expected to be identical. Evidently, this procedure allows us to get rid of the divergent selfenergy contributions and uniquely fixes the renormalization constants $c\left(\mu_{q}\right)$ and $c^{\prime}\left(\mu_{q}\right)$.

The renormalized $\Omega_{\bar{q} q}\left(r, \mu_{q}\right)$ is plotted in Fig. 1 for different values of the chemical potential. One can observe that $\Omega_{\bar{q} q}\left(r, \mu_{q}\right)$ shows the plateau already at $\mu=447 \mathrm{MeV}$. This happens because of the string breaking phenomenon, which for $\mu=447 \mathrm{MeV}$ takes place at $r \sim 0.5 \mathrm{fm}$. Of course string breaking occurs also for smaller chemical potentials, but we do not observe it, as it takes place beyond available distances. From Fig. 1 one also sees that the larger the chemical potential, the smaller the distance at which the string breaking takes place. 


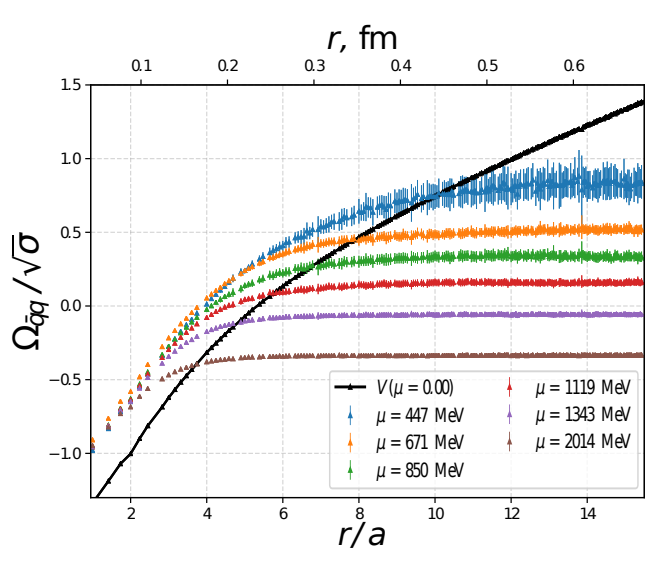

Figure 1: The $\Omega_{\bar{q} q}$ as a function of distance for few values of the chemical potential under study. The black curve is the potential of the static quarkantiquark pair at zero density and temperature. Note the absence of a Coulombic small distance regime, due to smearing.

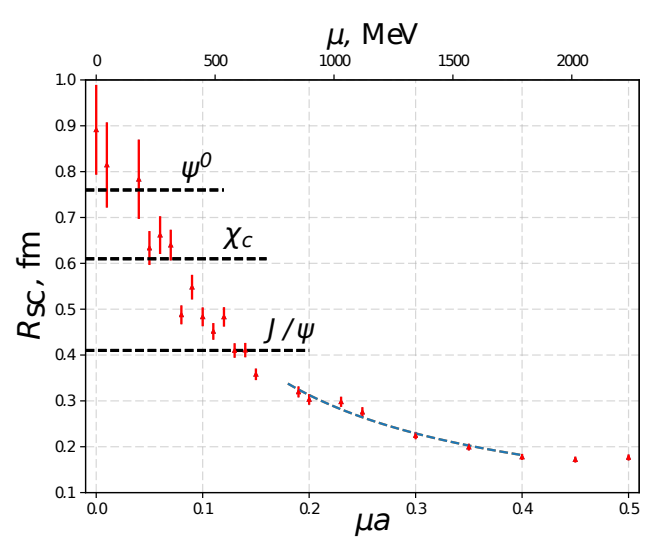

Figure 2: The screening length calculated from equation (3.1) as a function of chemical potential. Black dashed lines represent mean squared radii $\sqrt{\left\langle r^{2}\right\rangle}$ of charmonia. The blue dashed line is the description of the screening length $R_{s c}$ by the Debye screening formula (4.2).

\section{String breaking in dense quark matter}

To study the string breaking phenomenon we introduce the screening length $R_{s c}$ which can be calculated from the solution of the equation [1]

$$
V_{\mu=0}\left(R_{s c}\right)=\Omega_{\bar{q} q}\left(\infty, \mu_{q}\right),
$$

where $V_{\mu=0}(r)$ is the static potential at zero density. For $\Omega_{\bar{q} q}\left(\infty, \mu_{q}\right)$ we take the grand potential calculated from the renormalized Polyakov loop measured on the lattice, the results are shown in Fig. 2. This plot tells us that the larger is $\mu_{q}$ the smaller is the string breaking distance.

In order to understand this behaviour, let us recall that in three-color QCD the string breaking phenomenon can be explained by the possibility to break the string between static quarks by a quark-antiquark pair created from vacuum. If the length of the string is larger than the critical one it becomes energetically favorable to break the string and form two heavy-light meson instead of increasing the length of the string. In dense two-color QCD in addition to the possibility to break the string by quark-antiquark pairs it becomes possible to break the string by two quarks. As the result of this phenomenon, after the string has been broken, one ends up with a heavy-light meson and one heavy-light diquark. Due to confinement, the two quarks have to be extracted from some hadron. The two-color baryon - the scalar diquark is a good candidate for such a hadron. Indeed at nonzero $\mu_{q}$ the scalar diquark is a lightest state in the system. This picture is supported at large $\mu_{q}$ in the BCS phase, where one has a Fermi sphere with radius $\mu_{q}$. Evidently one cannot break the string by taking two quarks deep inside the Fermi sphere, since in that case, the quarks which break the string due to the interactions have to move from one point of the Fermi sphere to some other point inside the Fermi sphere. However, all points inside the Fermi sphere are occupied. So, the only possibility to break the string is to take two quarks close to the Fermi surface. In the confined phase, quarks on the Fermi surface are condensed as diquarks. Thus we again confirm the picture that two quarks, which break the string, can be taken from the available diquarks. 
If one increases the chemical potential then at some density $R_{s c}$ becomes so small that the string cannot be created, i.e. at the instant of creation it will be immediately broken by the twocolor baryons - diquarks. This is our hypothesis of the deconfinement mechanism in two-color dense quark matter. It is not clear how to find unambiguously the distance at which the string ceases to be stable. Using figure 2 one can infer that the interactions in this interval are screened, as the chemical potential is within $\mu_{q} \in(900,1300) \mathrm{MeV}$ and which agrees with the position of the confinement/deconfinement transition observed in [14].

In addition to the $R_{s c}$ in figure 2 we plot the average heavy quarkonia $J / \Psi, \chi_{c}, \psi^{\prime}$ radii which where estimated in Appendix B of [16] within a simple potential model. It is clear that if the screening length is close to the heavy quarkonium radius this state is considerably modified by dense quark matter. From figure 2 one sees that the heaviest state the $\psi^{\prime}$ due to its rather large radius should be considerably modified at nonzero density before the transition to BEC phase. The $\chi_{c}$ meson will instead be modified in the BEC phase. Finally we predict that the $J / \Psi$ meson will be modified in dense quark matter but before the deconfiment region. Notice, however, that if the radius of a charmonium equals to the $R_{s c}$ at some density $n_{0}$, the dissociation of this charmonium will take place at densities larger than $n_{0}$.

\section{Debye screening in dense quark matter}

In the region $\mu_{q}>900 \mathrm{MeV}$ the system under study undergoes transition from the confined to the deconfined phase (see Fig. 3). In the deconfined phase the contribution of the string state is absent and one may attempt to describe the screening in a dense quark-gluon plasma via an analogy with the Abelian theory, i.e. purely Coulombic Debye screening. The scale of the Debye screening in perturbation theory is denoted by the Debye mass, which to one-loop order (for the $N_{c}=2$ ) reads

$$
m_{D}^{2}\left(\mu_{q}\right)=\frac{4}{\pi} \alpha_{s}\left(\mu_{q}\right) \mu_{q}^{2}
$$

To describe the results for the $R_{s c}$ introduced in the previous section it is reasonable to assume that the screening length is inversely proportional to $m_{D}\left(\mu_{q}\right)$, thus we fit our data by the formula

$$
R_{s c}=\frac{1}{A m_{D}\left(\mu_{q}\right)}
$$

where the $A$ is the fit parameter. The data is fitted in the region $\mu_{q} \in(900,1800) \mathrm{MeV}$ with the use of a two-loop approximation for $\alpha_{s}\left(\mu_{q}\right)$ with $N_{f}=N_{c}=2$. The fit describes our data well $\left(\chi^{2} / d o f \simeq 0.8\right)$ and the best fit parameters are $A=1.4 \pm 0.4, \Lambda=140 \pm 80 \mathrm{MeV}$. In the region $\mu_{q}>1800 \mathrm{MeV}$ the data cannot be described by the formula (4.2).

Let us study how the Debye screening phenomenon manifests itself in the large distance behavior $\left(r \mu_{q} \gg 1\right)$ of the grand potential. In this case the dominant scale is the chemical potential, i.e. the running coupling constant depends only on $\mu_{q}: g\left(r, \mu_{q}\right)=g\left(\mu_{q}\right)$. For sufficiently large density one can apply perturbation theory to calculate grand potentials. Perturbatively the grand potential $\Omega_{\bar{q} q}\left(r, \mu_{q}\right)$ is determined by two-gluon exchange and it is rapidly decreasing with distance function. Contrary to $\Omega_{\bar{q} q}\left(r, \mu_{q}\right)$ the color singlet grand potential $\Omega_{1}\left(r, \mu_{q}\right)$ is determined by one-gluon exchange. 


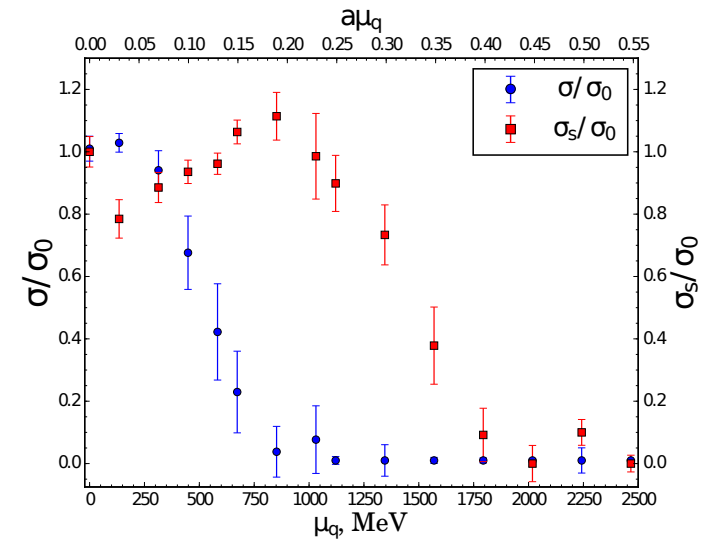

Figure 3: String tension $\sigma$ and spatial string tension $\sigma_{s}$ between quark-antiquark pair as functions of the chemical potential.

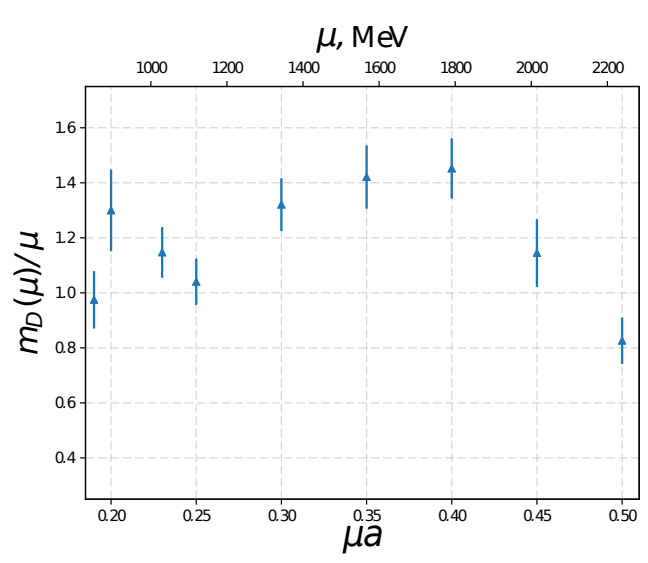

Figure 4: The ratio $m_{D} / \mu$ as a function of the chemical potential calculated from the fit of lattice data by formula (4.3).

In this study we consider only $\Omega_{1}\left(r, \mu_{q}\right)$, whose leading order contribution has the form

$$
\Omega_{1}\left(r, \mu_{q}\right)=\Omega_{1}\left(\infty, \mu_{q}\right)-\frac{3}{4} \frac{\alpha_{s}\left(\mu_{q}\right)}{r} e^{-m_{D} r},
$$

where $m_{D}$ is the Debye mass given by the expression (4.1). It tells us that due to Debye screening at sufficiently large distance the expression $\left(\Omega_{1}\left(\infty, \mu_{q}\right)-\Omega_{1}\left(r, \mu_{q}\right)\right) r$ is an exponentially decreasing function of the distance. We indeed observe such exponential decrease at large distance starting from $\mu_{q} \sim 850 \mathrm{MeV}$, what confirms Debye screening phenomenon in deconfined dense quark matter. The deviation from a purely Coulombic Debye-like behavior at intermediate distances may be related to the remnants of the string, which is not perfectly screened.

Further we fit our data in the deconfinement phase for $\Omega_{1}\left(r, \mu_{q}\right)$ at sufficiently large $r$ by the formula (4.3). The results for $m_{D} / \mu_{q}$ as a function of the chemical potential are shown in figure 4. From figure 4 it is seen that the dependence of the Debye mass on the chemical potential is $m_{D} \sim \mu_{q}$. Due to large uncertainties of the calculation, we are not able to resolve the running of the coupling constant with $\mu_{q}$. The running coupling is constant within the uncertainty of the calculation for $\mu_{q}<1800 \mathrm{MeV}$ and it starts to drop in the region $\mu_{q}>1800 \mathrm{MeV}$. In addition one can expect that the one-loop formula for the Debye mass (4.1) is considerably modified by higher order radiative corrections. In the deconfined phase at finite temperature and zero density one also obtains a large coupling constant (see e.g. [2, 3]).

\section{Conclusion and discussion}

The aim of the present paper was the study of the interaction between a static quark-antiquark pair in two-color dense quark matter. We studied the screening phenomenon in dense SU(2) QCD. In the confined phase, the screening length is determined by the string breaking length, whereas in the deconfined phase $R_{s c}$ is determined by the Debye screening phenomenon. The result of the calculation of the screening length shows that, consistent with intuition, the larger the chemical potential is, the smaller is the string breaking distance. We believe that the decrease of the string 
breaking distance with density can be attributed to the following string breaking mechanism in dense matter. In dense two-color QCD, in addition to the possibility to break the string by a quarkantiquark pair, it becomes possible to break the string by two quarks which can be extracted from a two-color baryon - the scalar diquark. As the result of this phenomenon, after the string breaking one ends up with one heavy-light meson and one heavy-light diquark. Lattice studies show [17] that in the region $\mu_{q}>m_{\pi} / 2$ the scalar diquark condensate increases with the chemical potential, i.e. it becomes easier to find two quarks and to break the string. At some density $R_{s c}$ becomes so small that the string cannot be created at all. Once created it will be immediately broken by the two-color baryons - the scalar diquarks. This is our hypothesis of the deconfinement mechanism in two-color dense quark matter.

The behavior of the string breaking distance in dense matter and the deconfinement mechanism are not specific only for two-color QCD. We believe that a similar process can be realized in SU(3) QCD with the difference that one has to replace two-quark baryon in SU(2) by three-quark baryon in SU(3). In particular, one can expect that the screening length, which has the same definition as in two-color QCD, is decreasing function of the chemical potential. For three colors this behavior can be explained as follows: at non-zero chemical potential one has a nonzero baryon density in the system. Baryons which form this density can break the string, splitting it into one quark and one diquark. Notice that one does not need additional energy to create the baryon since the baryons are already present, due to the nonzero chemical potential. After the string breaking one has one heavy-light meson and heavy-light baryon. Finally, the larger the chemical potential is, the larger is the number of baryons which can break the string, i.e. the string breaking distance is a decreasing function of the chemical potential.

We calculated the Debye mass and the coupling constant for various chemical potentials. The coupling constant extracted in this way takes on values $\alpha_{s} \sim 1$, which tells us that despite the large baryon density, the system remains strongly coupled. We found that the region $\mu<2000 \mathrm{MeV}$ physically differs from the region $\mu_{q}>2000 \mathrm{MeV}$, which manifests itself in different behavior of the following observables: the Polyakov line, the grand potential, the screening length $R_{s c}$ and the Debye mass. While we do not yet fully understand the physics, which is responsible for this behavior, one possibility is that the value of the chemical potential $\mu_{q} \sim 2000 \mathrm{MeV}$ is exceptional since it divides the region with finite spatial string tension from that where it vanishes. This may imply that the point $\mu_{q} \sim 2000 \mathrm{MeV}$ separates systems with and without magnetic screening.

\section{Acknowledgments}

V. V.B. acknowledges the support from the BASIS foundation. N. Yu. A. acknowledges the support from the BASIS foundation and FAIR-Russia Research Center. The work of A. Yu. K. was supported by FAIR-Russia Research Center and RFBR grant 18-32-00071. The work of A. A. N. was supported by RFBR grant 18-32-00104. V. G. B. acknowledges the support by RFBR grant 16-02-01146. A. R. acknowledges partial support by the DFG funded collaborative research center SFB1225 "ISOQUANT". This work has been carried out using computing resources of the federal collective usage center Complex for Simulation and Data Processing for Mega-science Facilities at NRC "Kurchatov Institute", http: / / ckp. nrcki . ru/. In addition, the authors used the cluster of the ITEP and the supercomputer of Joint Institute for Nuclear Research "Govorun". 


\section{References}

[1] O. Kaczmarek, F. Karsch, P. Petreczky and F. Zantow, Heavy quark anti-quark free energy and the renormalized Polyakov loop, Phys. Lett. B543 (2002) 41 [hep-lat/ 0207002 ].

[2] O. Kaczmarek, F. Karsch, F. Zantow and P. Petreczky, Static quark anti-quark free energy and the running coupling at finite temperature, Phys. Rev. D70 (2004) 074505 [hep-lat / 0406036 ].

[3] O. Kaczmarek and F. Zantow, Static quark anti-quark interactions in zero and finite temperature QCD. I. Heavy quark free energies, running coupling and quarkonium binding, Phys. Rev. D71 (2005) 114510 [hep-lat/0503017].

[4] Y. Maezawa, T. Umeda, S. Aoki, S. Ejiri, T. Hatsuda, K. Kanaya et al., Application of fixed scale approach to static quark free energies in quenched and 2+1 flavor lattice QCD with improved Wilson quark action, Prog. Theor. Phys. 128 (2012) 955 [1112.2756].

[5] SESAM collaboration, G. S. Bali, H. Neff, T. Düessel, T. Lippert and K. Schilling, Observation of string breaking in QCD, Phys. Rev. D71 (2005) 114513 [hep-lat/ 0505012 ].

[6] TUMQCD collaboration, A. Bazavov, N. Brambilla, P. Petreczky, A. Vairo and J. H. Weber, Color screening in (2+1)-flavor QCD, Phys. Rev. D98 (2018) 054511 [1804.10600].

[7] C. S. Fischer, L. Fister, J. Luecker and J. M. Pawlowski, Polyakov loop potential at finite density, Phys. Lett. B732 (2014) 273 [1306.6022].

[8] N. O. Agasian, M. S. Lukashov and Yu. A. Simonov, Nonperturbative SU(3) thermodynamics and the phase transition, Eur. Phys. J. A53 (2017) 138 [1701.07959].

[9] M. Andreoli, C. Bonati, M. D’Elia, M. Mesiti, F. Negro, A. Rucci et al., Gauge-invariant screening masses and static quark free energies in $N_{f}=2+1$ QCD at nonzero baryon density, Phys. Rev. D97 (2018) 054515 [1712.09996].

[10] J. B. Kogut, M. A. Stephanov and D. Toublan, On two color QCD with baryon chemical potential, Phys. Lett. B464 (1999) 183 [hep-ph/9906346].

[11] J. B. Kogut, M. A. Stephanov, D. Toublan, J. J. M. Verbaarschot and A. Zhitnitsky, QCD - like theories at finite baryon density, Nucl. Phys. B582 (2000) 477 [hep-ph/ 0001171$].$

[12] D. T. Son and M. A. Stephanov, QCD at finite isospin density, Phys. Rev. Lett. 86 (2001) 592 [hep-ph/0005225].

[13] O. Janssen, M. Kieburg, K. Splittorff, J. J. M. Verbaarschot and S. Zafeiropoulos, Phase Diagram of Dynamical Twisted Mass Wilson Fermions at Finite Isospin Chemical Potential, Phys. Rev. D93 (2016) 094502 [1509.02760].

[14] V. G. Bornyakov, V. V. Braguta, E. M. Ilgenfritz, A. Yu. Kotov, A. V. Molochkov and A. A. Nikolaev, Observation of deconfinement in a cold dense quark medium, JHEP 03 (2018) 161 [1711.01869].

[15] S. Nadkarni, Nonabelian Debye Screening. 1. The Color Averaged Potential, Phys. Rev. D33 (1986) 3738.

[16] N. Yu. Astrakhantsev, V. G. Bornyakov, V. V. Braguta, E. M. Ilgenfritz, A. Yu. Kotov, A. V. Molochkov et al., Lattice study of static quark-antiquark interactions in dense quark matter, 1808.06466 .

[17] V. V. Braguta, E. M. Ilgenfritz, A. Yu. Kotov, A. V. Molochkov and A. A. Nikolaev, Study of the phase diagram of dense two-color QCD within lattice simulation, Phys. Rev. D94 (2016) 114510 [1605.04090]. 